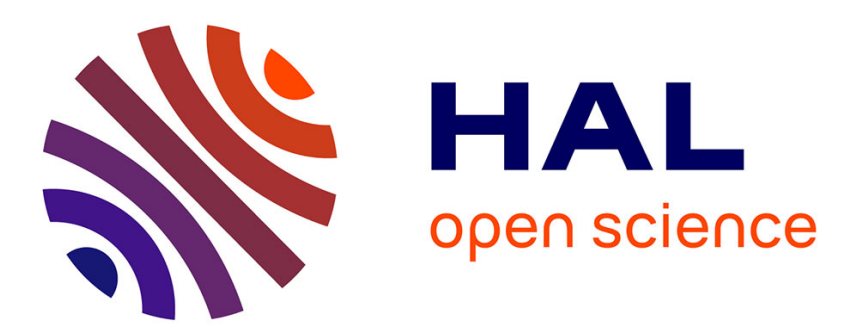

\title{
Étude paléontologique du Locus III de la Grotte de l'Eglise (Dordogne). Gisement ante Würmien à Bouquetin
}

Christophe Griggo, Griggo Christophe Étude

\section{- To cite this version:}

Christophe Griggo, Griggo Christophe Étude. Étude paléontologique du Locus III de la Grotte de l'Eglise (Dordogne). Gisement ante Würmien à Bouquetin. PALEO : Revue d'Archéologie Préhistorique, 1992, 4, pp.35 - 48. 10.3406/pal.1992.1192 . hal-03163669

\section{HAL Id: hal-03163669 \\ https://hal.science/hal-03163669}

Submitted on 9 Mar 2021

HAL is a multi-disciplinary open access archive for the deposit and dissemination of scientific research documents, whether they are published or not. The documents may come from teaching and research institutions in France or abroad, or from public or private research centers.
L'archive ouverte pluridisciplinaire HAL, est destinée au dépôt et à la diffusion de documents scientifiques de niveau recherche, publiés ou non, émanant des établissements d'enseignement et de recherche français ou étrangers, des laboratoires publics ou privés. 


\title{
Étude paléontologique du Locus III de la Grotte de l'Eglise (Dordogne). Gisement ante Würmien à Bouquetin
}

In: Paléo. N. 4,1992. pp. 35-48.

\begin{abstract}
Résumé
Jusqu'à ce jour, la Grotte de l'Eglise, dans la vallée du Céou, se caractérisait d'un point de vue paléontologique par l'association Canis etruscus, Ursus deningeri, Hemitragus bonali datée du Mindel supérieur. Le Locus m inférieur de cette même grotte a livré une faune différente dont Tige serait plus récent II n'y a pas de reste de Canidé et le Caprine présent est un Bouquetin. Une grande partie de ce travail est consacrée à la discrimination Hemitragus - Capra. La détermination des autres taxons est justifiée par la description de quelques éléments caractéristiques. L'étude se termine par une interprétation chronologique de l'association mammalienne ; la série pouvant être datée de la fin du complexe rissien (stade isotopique 6).
\end{abstract}

\section{Abstract}

Previous studies of the grotte de l'Eglise, in the Céou valley, was paleontologically caracterized by the association Canis etruscus, Ursus deningeri, Hemitragus bonali dated from the upper Mindel. The lower Locus IE of the same cave is probably more recent. There is no Canidae : the Caprinae is represented by lbex. Ursus deningeri is present. This study deals in taxonimic discrimination of caracteristic bones and chronological interpretation of the mammals association which could be dated by the end of Riss glaciation (isotopic stage 6).

Citer ce document / Cite this document :

Griggo Christophe. Étude paléontologique du Locus III de la Grotte de I'Eglise (Dordogne). Gisement ante Würmien à Bouquetin. In: Paléo. N. 4,1992. pp. 35-48.

doi : $10.3406 /$ pal.1992.1192

http://www.persee.fr/web/revues/home/prescript/article/pal_1145-3370_1992_num_4_1_1192 


\title{
ETUDE PALEONTOLOGIQUE DU LOCUS III DE LA GROTTE DE L'EGLISE (Dordogne). \\ Gisement anté würmien à Bouquetin
}

\author{
Christophe GRIGGO*
}

\begin{abstract}
Résume :
Jusqu'a ce jour, la Grotte de l'Eglise, dans la vallée du Céou, se caractérisait d'un point de vue paléntologique par l'association Canis etruscus, Ursus deningeri, Hemitragus bonali daţe du Mindel supérieur. Le Locus III inférieur de cette même grotte a livré une faune différente dont líge serait plus récent. Il n'y a pas de reste de Canidé et le Capriné présent est un Bouquetin. Une grande partie de ce travail est consacrée à la discrimination Hemitragus - Capra. La détermination des autres taxons est justifíé par la description de quelques ééments caractéristiques. L'étude se termine par une interprétation chronologique de l'association mammalienne ; la série pouvant être datée de la fin du complexe rissien (stade isotopique 6).

Abstract :

Previous studies of the grote de l'Eglise, in the C6ou valley, was paleontologically caracterized by the association Canis etruscus, Ursus deningeri, Hemitragus bonali dated from the upper Mindel. The lower Locus III of the same cave is probably more recent. There is no Canidae : the Caprinae is represented by Ibex. Ursus deningeri is present. This study deals in taxonimic discrimination of caracteristic bones and chronological interpretation of the mammals association which could be dated by the end of Riss glaciation (isotopic stage 6).
\end{abstract}

La grotte de l'Eglise se situe dans la commune de Cénac-et-Saint-Julien, au lieu dit "La Falaise du Conte", sur la rive droite de la vallée du Céou, affluent de la rive gauche de la Dordogne. Cette grotte appartient à un complexe karstique creusé dans le calcaire jaunâtre du Coniacien qui constitue une façade rocheuse où l'on observe plusieurs entrées de grottes numérotées de I à XX par J. Lachastre. La Grotte de l'Eglise correspond au numéro XIII.

Le remplissage de cette cavité a déjà fait l'objet d'une étude paléontologique par F. Prat (H. Laville et al 1972). Le matériel osseux dont il est question dans ce travail provient essentiellement de la galerie principale et du diverticule I (Fig. 1). Il appartient à une faune du Mindel supérieur comprenant notamment Ursus deningeri, Canis etruscus et Hemitragus bonali.

Les ossements qui ont retenu notre attention ont été découverts dans le Locus III inférieur, sorte de diaclase s'ouvrant par un conduit étroit dans le diverticule I (Fig.2). Ils ont été recueillis en partie par le Docteur Malenfant qui les a confiés à l'Institut du Quaternaire de l'Université de Bordeaux I. D'autres pièces furent mises au jour lors de fouilles très limitćes effectuées par F. Prat en 1968. Ce dernier nous a aimablement communiqué quelques observations concernant les dépôts accessibles du locus III inférieur :

- la stratigraphie a été établie à partir d'une coupe de $40 \mathrm{~cm}$ de hauteur. F. Prat a distingue, de haut en bas, quatre couches : (Fig. 2 et 3 )

0 - Sédiment tassé par les visiteurs.

1 - Sable brunâtre à microfaune avec intercalation plus claire -1a.
2 - Sédiment sableux jaunâtre meuble.

3 - Sable induré : 2 à $6 \mathrm{~cm}$.

4 - Sable brun jaunâtre avec passées brunes. Cette couche est particulièrement épaisse vers la paroi : plus de $17 \mathrm{~cm}$.

La base de cette couche n'a pas été atteinte

- L'essentiel du matériel récolté par F. Prat provient de la couche 3 . Les pièces de la collection du Docteur Malenfant ont été récoltées en surface.

- Aucune corrélation stratigraphique n'a pu être établie avec les autres zones de la grotte fouillées par F. Prat.

- Il faut noter que tous les os que nous avons étudié ont une patine semblable comme s'ils provenaient de la même formation.

La liste des genres et des espèces déterminés est fournie dans le tableau I.

Nous pouvons noter dès maintenant que la seule considération de la présence de capra hircus cf. ibex permet de distinguer cet ensemble de ceux rencontrés dans d'autres parties de la grotte où selon F. Prat (in H. Laville et al. 1972) le seul Capriné présent est un Thar, Hemitragus bonali. Nous nous efforcerons donc, dans un premier temps, de nous assurer de l'identité du seul Ongulé bien représenté dans le Locus III inférieur puis nous déterminerons de façon aussi précise que possible les Mammifères qui lui sont associés, au moins en collection, peut-être en stratigraphie.

Nous ne pratiquerons pas d'analyse statistique des données, car n'ayant pas de séries d'os ou de dents

* Institut du Onaternaire, Université de Bordeavx 1, Av. des Facultés - 33405 TALENCE CEDEX. 
suffisamment riches, les résultats ne seraient pas significatifs.

\section{Capra hircus cf ibex.}

Les différences morphologiques entre Capra et Hemitragus, en ce qui concerne le squelette postcrânien et la denture, sột assez difficiles à saisir. Pour mener a bien notre étude, nous avons eu recours aux travaux de:

- R.P. Charles 1957.

- F. prat (in H. Laville et al. 1972).

- M.F. Bonifay 1974-75.

- E. Crégut-Bonnoure 1987.

Nous avons également examiné les restes d'Hemitragus provenant de la Grotte de l'Eglise étudiés par F. Prat, ainsi que ceux de la Grottre Vaufrey, étudiés par F. Delpech (1988). Les ossements de Bouquetin, qui nous ont servi de termes de comparaison, appartiennent aux collections de l'Institut du Quaternaire de l'Université de Bordeaux I.

\subsection{Les dents}

R.P. Charles (1957) et M.F Bonifay (1974-75) sont unanimes pour affirmer que la morphologie des dents jugales du Bouquetin est très proche de celle des dents jugales du Thar. La distinction entre ces deux espèces à partir des molaires isolées est donc délicate. R.P. Charles a bien établi quelques critères d'identification mais ils n'intéressent pas tous les éléments de la denture. Ainsi une P2 et une M2 de Capriné n'ont pu être determinées avec précision.

La M3 présente quant à elle des fossettes non ouvertes du côté lingual. Cette disposition est caractéristique du Bouquetin d'après R.P. Charles (1957 p. 139). En fait, ceci est inexact car, chez cet animal ; les fossettes s'ouvrent bien vers l'intérieur dans la partie supérieure de la couronne des molaires inférieures peu ou non usées. Nous n'observons pas cette dispositon sur notre spécimen, simplement car il est trop entamé par l'usure.

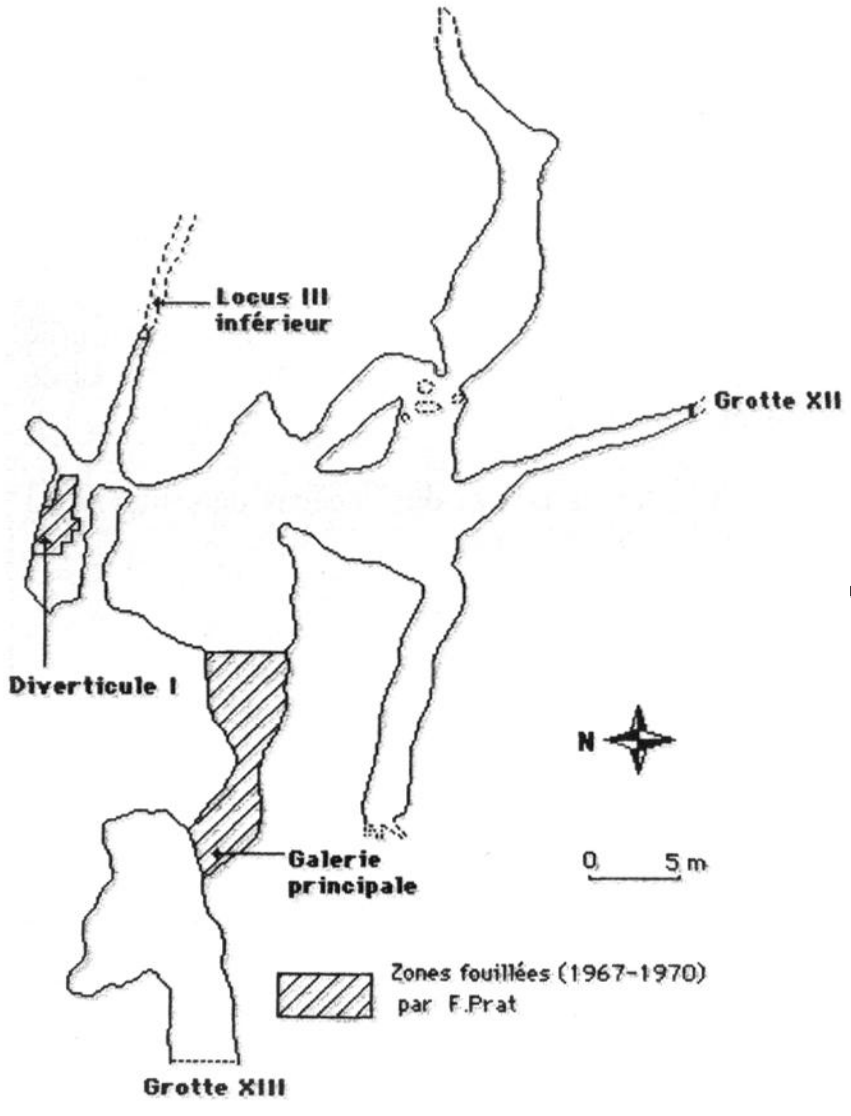

Fig.1 : Plan de la Grotte de l'Eglise à Cénac-etSaint-Julien (Dordogne) établi par le spéléo-club de Carbon-BlancC Gironde).

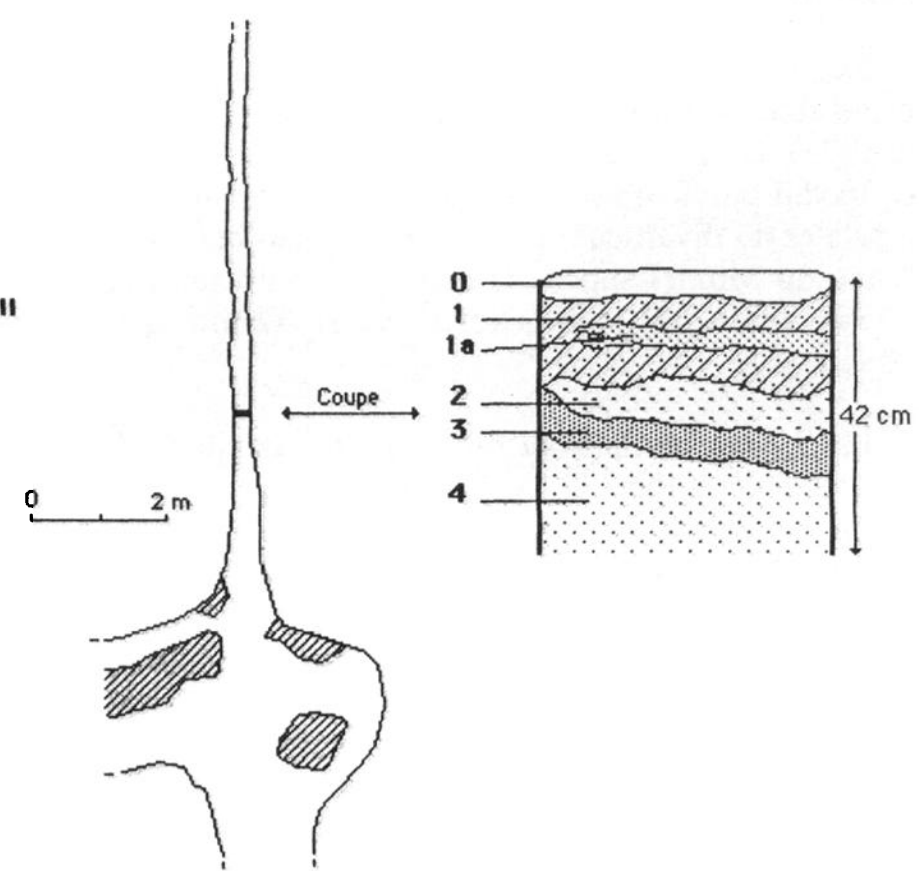

Fig. 2 : Plan du Locus III inférieur (d'après F. Prat).

Fig. 3 : Stratigraphie de la coupe du Locus III inférieur (d'après F. Prat). 


\begin{tabular}{|l|l|l|l|l|l|l|l|}
\hline Espèces & $\begin{array}{l}\text { Eléments } \\
\text { crâniens }\end{array}$ & $\begin{array}{l}\text { Squelette } \\
\text { axial+côte }\end{array}$ & $\begin{array}{l}\text { Membre } \\
\text { antérieur }\end{array}$ & $\begin{array}{l}\text { Membre } \\
\text { postérieur }\end{array}$ & Phalanges & $\begin{array}{l}\text { Nbre de } \\
\text { restes }\end{array}$ & $\%$ \\
\hline Panthera spelaea & 0 & 1 & 0 & 1 & 2 & 4 & 1,64 \\
\hline Lynx sp. & 0 & 0 & 0 & 1 & 0 & 1 & 0,41 \\
\hline Vulpes ou Alopex & 1 & 1 & 0 & 0 & 0 & 2 & 0,82 \\
\hline Meles sp. & 0 & 0 & 0 & 0 & 1 & 1 & 0,41 \\
\hline Ursus deningeri & 36 & 5 & 14 & 16 & 39 & 110 & 45,27 \\
\hline Ursus spelaeus & 2 & 0 & 3 & 1 & 0 & 6 & 2,47 \\
\hline Ursus arctos & 2 & 0 & 0 & 4 & 1 & 7 & 2,88 \\
\hline Capra hircus cf. ibex & 3 & 15 & 18 & 8 & 45 & 89 & 36,62 \\
\hline Rupicapra rupicapra ssp. & 0 & 0 & 1 & 0 & 0 & 1 & 0,41 \\
\hline Dicerorhinae indét. & 0 & 0 & 0 & 0 & 1 & 1 & 0,41 \\
\hline Oryctolagus cuniculus grenalensis & 0 & 0 & 0 & 17 & 4 & 21 & 8,64 \\
\hline Totaux & & & & & $\mathbf{2 4 3}$ & $\mathbf{9 9 , 9 8}$ \\
\hline
\end{tabular}

Tableau. I : Représentation des genres et espèces déterminés.

\begin{tabular}{|c|c|c|c|c|c|c|c|c|}
\hline $\begin{array}{l}\text { Mesures } \\
\text { en mm }\end{array}$ & $\begin{array}{r}\text { Bouquetin } \\
\text { Gr.de l'Eglise }\end{array}$ & \multicolumn{3}{|c|}{$\begin{array}{c}\text { Thar } \\
\text { Gr.de l'escale } \\
\text { Bonifay } 1974-75\end{array}$} & \multicolumn{4}{|c|}{$\begin{array}{c}\text { Bouquetin des Pyrénées } \\
\text { Malarnaud } \\
\text { Prat } 1972\end{array}$} \\
\hline L.T. & 201,0 & 210,0 & 220,6 & 191,8 & 199,5 & 222,5 & 225,5 & 195,5 \\
\hline D.T.prox. & 45,0 & 44,9 & 47,8 & 39,3 & 39,5 & 46,5 & 45,0 & 40,0 \\
\hline D.T.méd. & 28,0 & 27,4 & 27,5 & 22,7 & 26,0 & 31,5 & 32,5 & 23,5 \\
\hline D.T.dist. & 43,0 & 42,8 & 40,7 & 22,7 & 32,5 & 37,0 & 39,0 & 31,5 \\
\hline$\frac{\text { D.T.méd. } * 100}{\text { L.T. }}$ & 13,9 & 13,0 & 12,4 & 11,8 & 13,0 & 14,1 & 14,2 & 13,1 \\
\hline
\end{tabular}

Tableau II : Bouquetin et Thar - Dimensions du radius.

\subsection{Le radio-ulnaire. (Fig.4)}

Le radio-ulnaire provenant du Locus III inférieur est bien conservé. Radius et ulna sont intimement soudés dans la région proximale du radius ; à l'extrémité distale, ils sont, au contraire, indépendants l'un de l'autre : une fente profonde sépare l'apophyse styloïde de l'ulna du bord articulaire latéral du radius mais celle-ci est présente à la fois chez le Bouquetin et chez le Thar (F. Prat 1966 in Laville et al., op. cit.). F. Prat observe au contraire que le radius de Thar découvert dans la Grotte de l'Eglise, dans la couche 6 de la galerie principale, ne porte sur la face postérieure de son extrémité proximale aucune trace d'ulna adhérent. La pièce appartient pourtant à un animal adulte. La suture intime des deux os vers le sommet du radius pourrait donc bien être caractéristique des Bouquetins. Si ce critère de détermination est valable, ce qu'il n'est pas possible d'affirmer en raison de la pauvreté de notre documentation relative au Thar, le radius du Locus III inférieur serait celui d'un Bouquetin.
Selon F. Prat (op. cit), le bord postérieur de l'articulation proximale est moins élevé chez le Bouquetin que chez le Thar. En cela le radius du Locus III inférieur ne diffère pas de celui du Bouquetin. La tubérosité latérale sur laquelle s'insère le ligament latéral de l'articulation huméroradiale serait plus proéminente et en position plus haute chez le Bouquetin que chez le Thar (F. Prat, op. cit.). Là encore nous n'observons pas de différence entre notre spécimen et le radius de Bouquetin.

Les deux condyles articulaires de l'extrémité distale du radius sont obliques, comme il est de règle chez les Ruminants, mais chez le Bouquetin, et c'est le cas du radius du Locus III inférieur de la Grotte de l'Eglise, le condyle latéral en relation avec le semi-lunaire est très déprimé, alors que le condyle médial se révèle fortement proéminant vers le bas. Ceci entraine un fort décalage entre les saillies distales des deux condyles. Chez le Thar, ce décalage est relativement moins accentué (F. Prat in H. Laville et al., op.cit. p.105). 
(1)
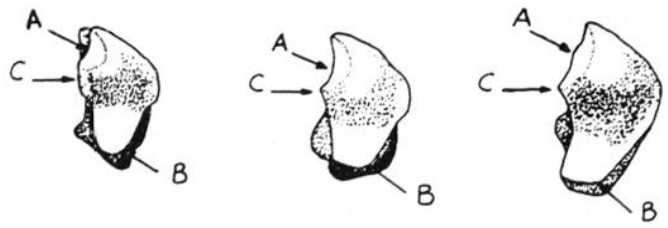

(2)<smiles>[2H]C1C=CC2CCCCC12</smiles><smiles>C1CC[P]CC1</smiles><smiles>[2H]C1CCCCC1</smiles>

(3)
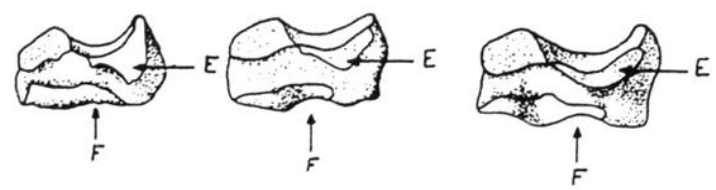

Fig. 5 : Scaphoide gauche - 1 : Vue proximale ; 2 : Vue palmaire ; 3 : Vue latérale.

Gr. de l'Escale : Thar ;

Valescure et Gr. de l'Eglise : Bouquetin.

(Gr. de l'Escale, Valescure : d'après E. CregutBonnoure)
(1)

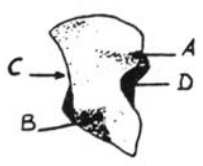

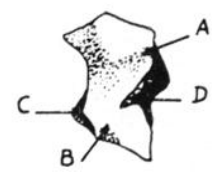

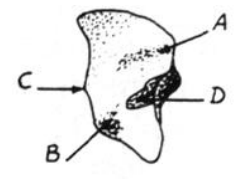

(2)
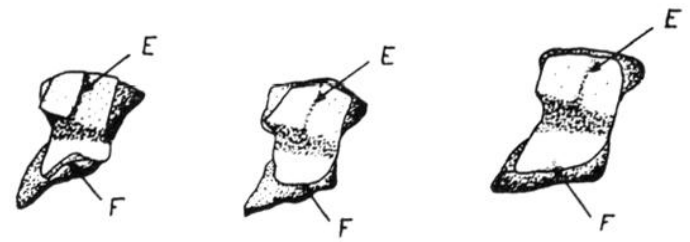

Fig. 6 : Semi-lunaire gauche -1 : Vue proximale, 2: Vue distale.

Gr. de l'Escale : Thar ; Valescure et Gr. de l'Eglise : Bouquetin.

(Gr. de l'Escale, Valescure : d'après E. CregutBonnoure).
Fig. 4 : Bouquetin - Radio-ulnaire droit - Gr. de l'Eglise. Locus III inf. 
Examiné en vue antérieure, le bord médial de la diaphyse du radius du Locus III inférieur présente une assez forte convexité chez le Bouquetin ; chez le Thar, d'après M.F. Bonifay (1974-75), il est rectiligne.

En outre, le radius que nous étudions est relativement trapu ; son indice de robustesse atteint 13,9 tandis que chez le Thar, il est généralement plus faible (M.F. Bonifay 1974-75) (Tabl. II).

Toutes les observations, qu'elles soient d'ordre morphologique ou biométrique, vont dans le sens d'une attribution du radius de Capriné du Locus III inférieur au Bouquetin.

\subsection{Le Basipode.}

En utilisant les critères de différenciation proposés par E. Crégut-Bonnoure (1987), les caractères ci-dessous énumérés, propres aux ossements de la Grotte de l'Eglise, sont ceux de Bouquetin. Nous avons représenté figures 5 à 12, les carpiens et tarsiens de Bouquetin de la grotte de l'Eglise et figuré, à gauche de chacun d'eux, l'os homologue de Thar et celui de Bouquetin.

\subsubsection{Le carpe.}

\section{Le scaphoïde (Fig. 5)}

* En vue proximale :

- face dorso-latérale en contact avec le semilunaire oblique par rapport à l'axe dorso-palmaire (Fig. 5, 1A).

- partie articulaire palmaire relativement large (Fig. 5, 1B).

- Formation d'un "pic" entre les deux facettes latérales (Fig. 5, 1C).

* En vue palmaire :

- bord proximal arrondi (Fig. 5, 2D).

* En vue latérale :

- surface articulaire en forme de croissant (Fig. 5, 3E).

- bord distal arrondi (Fig. 5, 3F).

\section{Le semi-lunaire (Fig. 6)}

* En vue proximale :

- crête dorso-médiale (Fig. 6, 1A) et crête latéropalmaire (Fig.6, 1B) convergentes vers le centre. - bord latéral dessinant deux concavités qui déterminent un angle aigu vers l'extérieur (Fig. 6, 1c). - échancrure synoviale médiale très profonde (Fig.6,1D).
* En vue distale :

- crête de séparation des deux facettes articulaires dorsales peu marquée (Fig.6, 2E).

- bord articulaire palmaire sub-rectiligne (Fig.6, 2F);

Le Pyramidal (Fig. 7)
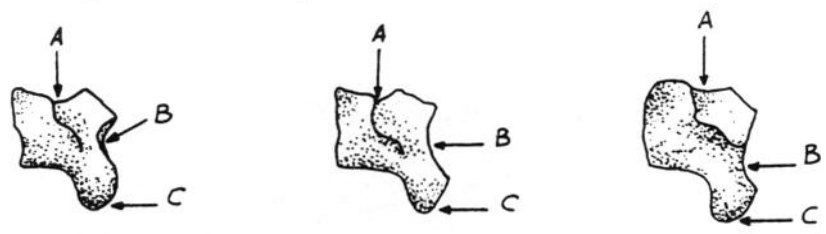

Fig.7 : Pyramidale gauche - Vue latérale. Gr.de l'Escale: Thar : Valescure et Gr.de l'Eglise: Bouquetin. (Gr.del'Escale,Valescure : d'après E.Cregut-bonnoure)

* En vue latérale :

- surface articulaire proximale répondant à l'ulna, peu concave (Fig. 7, A).

- surface articulaire répondant au pisiforme, relativement allongée et plane (Fig.7, B).

- tubercule disto-palmaire légèrement tronqué postérieurement (Fig. 7,C).

Le pisiforme. (Fig.8)
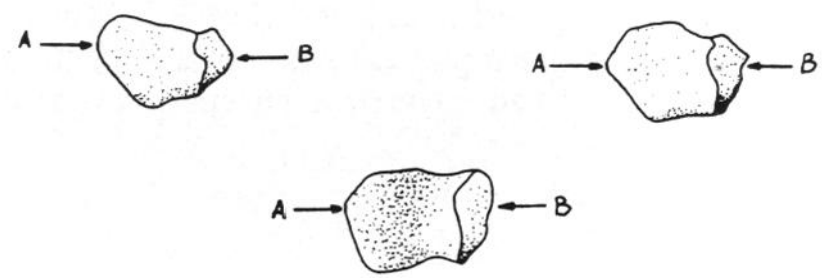

Fig.8 : Pisiforme gauche - Vue médiale.

Gr.de l'Escale: Thar ; Valescure et Gr.de l'Eglise: Bouquetin (Gr.del'Escale,Valescure : d'après E.Cregut-bonnoure)

* En vue médiale :

- bord palmaire formant un angle obtus (Fig. 8,A).

- surface articulaire répondant au pyramidal relativement allongée et plane (Fig. 8, B).

Le capitato-trapézoïde (Fig. 9)
(1)

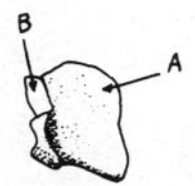

(2)

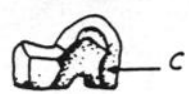

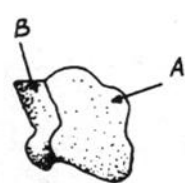
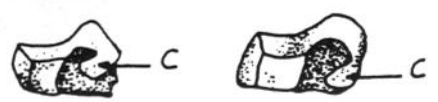

Fig.9 : Capitato-trapézoīde gauche - 1: Vue proximale, 2: Vue latérale. Gr.de l'Escale: Thar; Valescure et Gr.de l'Eglise: Bouquetin. (Gr.del'Escale,Valescure : d'après E.Cregut-honnoure)

* En vue proximale : 
- reliefs de la surface articulaire médiane répondant au scaphoïde relativement accusés (Fig. 9, 1a).

- crête séparant les facettes articulaires en relation l'une avec le scaphoïde, l'autre avec le semi-lunaire formant une angulation nette (Fig.9, 1B).

* En vue latérale :

- surface articulaire palmaire s'incurvant de l'avant vers l'arrière (Fig. 9, 2C);

\section{L'unciforme. (Fig.10)}
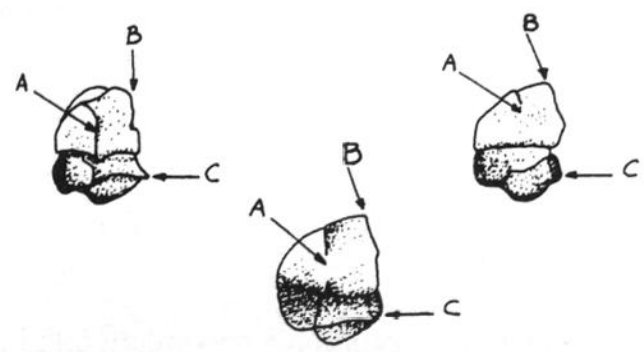

Fig.10 : Unciforme gauche - Vue proximale

Gr.de l'Escale: Thar ; Valescure et Gr.de l'Eglise: Bouquetin. (Gr.del'Escale, Valescure : d'après E.Cregut-honnoure)

* En vue proximale :

- facettes articulaires répondant au semi-lunaire et au pyramidal séparées par une crête qui s'estompe vers l'arrière (Fig. 10,A);

- bords dorsal et médial de la facette articulaire médiale formant un angle pratiquement droit (Fig. 10, B);

- facette articulaire palmaire de la face médiale oblique vers le haut et visible en vue proximale (Fig.10,C).

\subsubsection{Le tarse.}

Le talus (fig. 11)

(1)
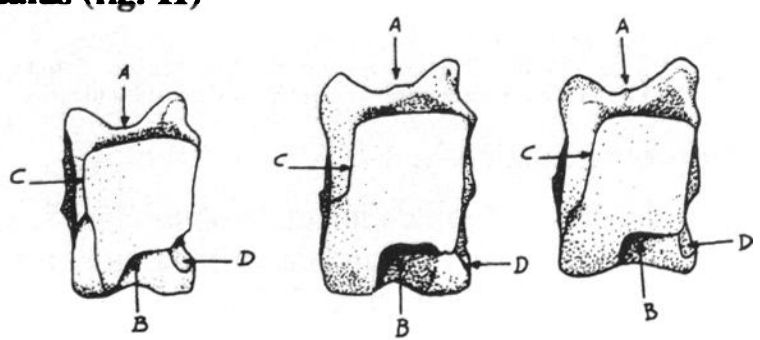

(2)
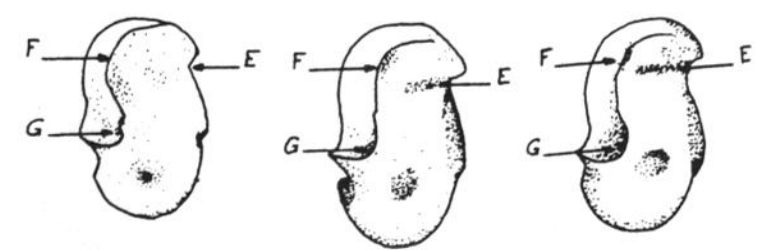

Fig.11 : Talus droit - 1: Vue plantaire, 2: Vue médiale. Gr.de l'Escale: Thar ; Valescure et Gr.de l'Eglise: Bouquetin. (Gr.del'Escale,Valescure : d'après E.Cregut-honnoure)

* En vue plantaire :
- gorge située entre les deux lèvres supérieures relativement large présentant au centre une petite crête (Fig. 11, 1A).

- surface de la trochlée calcanéenne séparée de celle de la trochlée distale par un ressaut important, souvent nettement anguleux (Fig. 11, 1B).

- pas d'échancrure synoviale dans cette zone (Fig.11,1D).

- bord médial de la trochlée plantaire en position nettement interne (Fig.11,1C).

* En vue médiale :

- légère dépression transversale à la base de la trochlée médio-plantaire (Fig.11, 2E).

- levvre articulaire proximo-médiale présentant un bord plantaire peu saillant (Fig.11, 2F) et une base très déprimée (Fig.11, 2G).

\subsubsection{Le métacarpe. (Fig.12).}

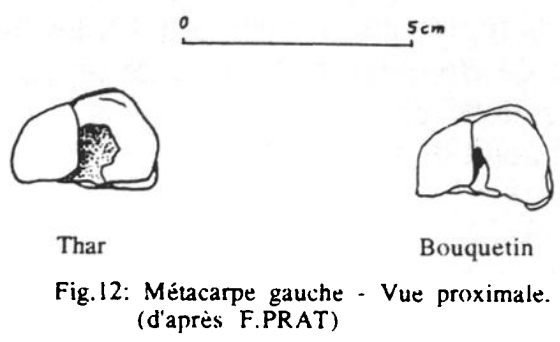

F. Prat (in H. Laville et al 1972, p.107) constate que chez le Bouquetin, au niveau de l'articulation proximale, le bord latéral de la facette répondant à l'unciforme décrit un arc de cercle plus régulier chez le Thar que chez le Bouquetin (Fig. 12,A). Il observe le contraire en ce qui concerne le bord médial de la facette répondant au capitato-trapézoïde (Fig.12,B). Dans la région distale, il n'a pas relevé de caractères distinctifs.

Les 4 métacarpes complets provenant du Locus III inférieur présentent, dans leur région proximale, les caractères propres aux os canons antérieurs du Bouquetin.

Les valeurs de l'indice de robustesse (tabl. III) calculees pour ces pièces ne sont pas discriminantes car les intervalles de variation de cet indice relatifs à l'un ou à l'autre genre se chevauchent nettement (M.F. Bonifay, 1974-75, p. 252). En revanche, la valeur de l'indice (largeur transversale proximale / Longueur totale) $\times 100$ (tabl.III) rapproche nos pièces des métacarpes de Bouquetin. En effet cet indice égale 24,1 en moyenne chez le Bouquetin (24,05 pour le spécimen du Locus III inférieur) et 23,6 chez le thar, calculs effectués à partir des données métriques publiés par M.F. Bonifay (op. cit.). 


\begin{tabular}{|c|c|c|c|c|c|c|c|c|c|c|c|c|c|}
\hline \multirow[t]{2}{*}{$\begin{array}{l}\text { Mesures } \\
\text { en mm }\end{array}$} & \multicolumn{4}{|c|}{$\begin{array}{c}\text { Bouquetin } \\
\text { Gr.de l'Eglise }\end{array}$} & \multicolumn{3}{|c|}{$\begin{array}{c}\text { Capra ibex } \\
\text { L' Observatoire } \\
\text { BONIFAY } 1974\end{array}$} & \multicolumn{3}{|c|}{$\begin{array}{c}\text { Hemitragus } \\
\text { Gr. de l' Escale } \\
\text { BONIFAY } 1974\end{array}$} & \multicolumn{3}{|c|}{$\begin{array}{l}\text { Hemitragus } \\
\text { Hunasheim } \\
\text { DAXNER } 1966\end{array}$} \\
\hline & & & & & $\mathrm{n}$ & $\mathrm{X}$ & $\mathrm{s}$ & $\mathrm{n}$ & $\mathrm{X}$ & s & $n$ & $\mathrm{X}$ & $\mathrm{s}$ \\
\hline LT & 139 & 139 & 135 & 139 & 26 & 142,8 & 1,25 & 25 & 139,9 & 6,54 & 16 & 145,1 & 3,91 \\
\hline DT.p & 32 & 34 & 33 & 34 & 26 & 34,6 & 3,45 & 38 & 32,6 & 0,34 & 16 & 33,8 & 1,33 \\
\hline DT.méd. & 21 & 22 & 21 & 22 & 26 & 24,2 & 2,58 & 24 & 22,3 & 0,28 & 16 & 23,1 & 1,01 \\
\hline DT.dist. & 37 & 37 & 37 & 40 & 26 & 39,6 & 4,10 & 21 & 37,8 & 2,15 & 15 & 38,7 & 1,33 \\
\hline $\begin{array}{l}\text { DT.méd. } * 100 \\
\text { LT }\end{array}$ & 15 & 15,8 & 15,5 & 15,8 & 26 & 16,8 & 1,03 & 24 & 15,8 & 0,72 & 16 & 15,9 & 0,66 \\
\hline$\frac{\text { DT.prox } * 100}{\text { LT }}$ & 23 & 24,4 & 24,4 & 24,4 & 26 & 24,1 & 1,64 & 21 & 23,6 & 0,75 & 16 & 23,1 & 0,87 \\
\hline
\end{tabular}

Tableau III : Bouquetin et Thar - Dimensions du métacarpe.

\subsubsection{Les phalanges.}

* Les premières phalanges. (fig.13)

Sur le bord dorsal de l'articulation proximale, la surface articulaire excentrique présente un angle rentrant prononce (Fig. 13a), tandis que le bord de la gorge médiane est convexe (Fig. 13,B).

* Les troisièmes phalanges (Fig. 14).

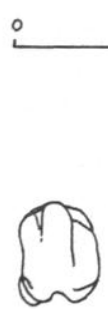

Thar Bouquetin

Fig.13: Première phalange (d'après F.PRAT)

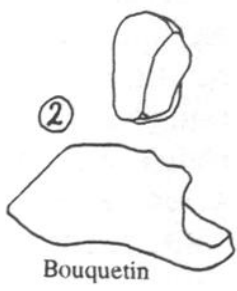

Fig.14 : Troisième phalange

1: articulation, 2: Face latérale (d'après F.PRAT)
La surface articulaire est large et profonde, plus large que chez Hemitragus. En vue latérale, l'extrémité antérieure apparaît aiguë et le bord de l'os est très irrégulier.

Selon F. prat (in H. laville et al. 1972, p. 107), ces dispositions seraient assez caractéristiques du Bouquetin.

\subsection{Conclusion}

Malgré l'absence de portions crâniennes ou de chevilles osseuses, nous pouvons affirmer, sans la moindre réserve, que le Capriné du Locus III inférieur de la Grotte de l'Eglise, ainsi que nous l'avons annoncé est bien un Bouquetin et non un Thar. La présence du genre Capra au sein de cette association méritera une attention spéciale lorsque nous tenterons de déterminer la position chronologique de cet ensemble faunique.

Actuellement, le Bouquetin est représenté, en Europe, par différentes formes géographiques. Celles-ci ont été regroupées par M. Clouet et P. Pfeffer (1979) au sein d'une seule espèce : Capra hircus, et correspondent donc à des sous-espèces géographiques.

En paléontologie, dans l'ćtat actuel des connaissances, la détermination de la sous-espèce ne peut se faire qu'à partir d'une cheville osseuse de corne bien conservée. En Europe occidentale, jusqu'ici, seules deux sous-espèces ont été identifiées : le Bouquetin des Alpes, Capra hircus ibex, dont les chevilles osseuses présentent une courbure régulière vers l'arrière et une section sub-triangulaire, et le Bouquetin des Pyrénées, Capra hircus pyrenaica, caractérisé par des appendices frontaux qui amorcent une torsion hélicoïdale et possèdent une section ovalaire (Voir références in C. Griggo, 1991)

En ce qui concerne la grotte de l'Eglise nous ne disposons d'aucun argument pour attribuer ces vestiges à la forme alpine (Capra hircus ibex) plutôt qu'à la forme pyrénéenne (Capra hircus pyrenaica). Une appartenance d̀ la première nous semble cependant plus vraisemblable étant donné que jusqu'ici tous les vestiges de Bouquetin identifiables avec précision provenant de gisements du Périgord ont été rapportés a Capra hircus ibex (F. Delpech, 1983 et J.L.Guadelli, 1987). 


\section{Les Mammiferes associés au Bouquetin dans le} Locus III inférieur.

\subsection{Panthera spelaea}

Cette espèce n'est représentée dans le Locus III inférieur de la grotte de l'Eglise que par trois pièces: - une $4^{\circ}$ ou $5^{\circ}$ vertèbre lombaire dont la face ventrale est endommagée.

- un os coxal droit.

- 2 phalanges proximales des doigts III ou IV.

\subsubsection{La vertèbre lombaire.}

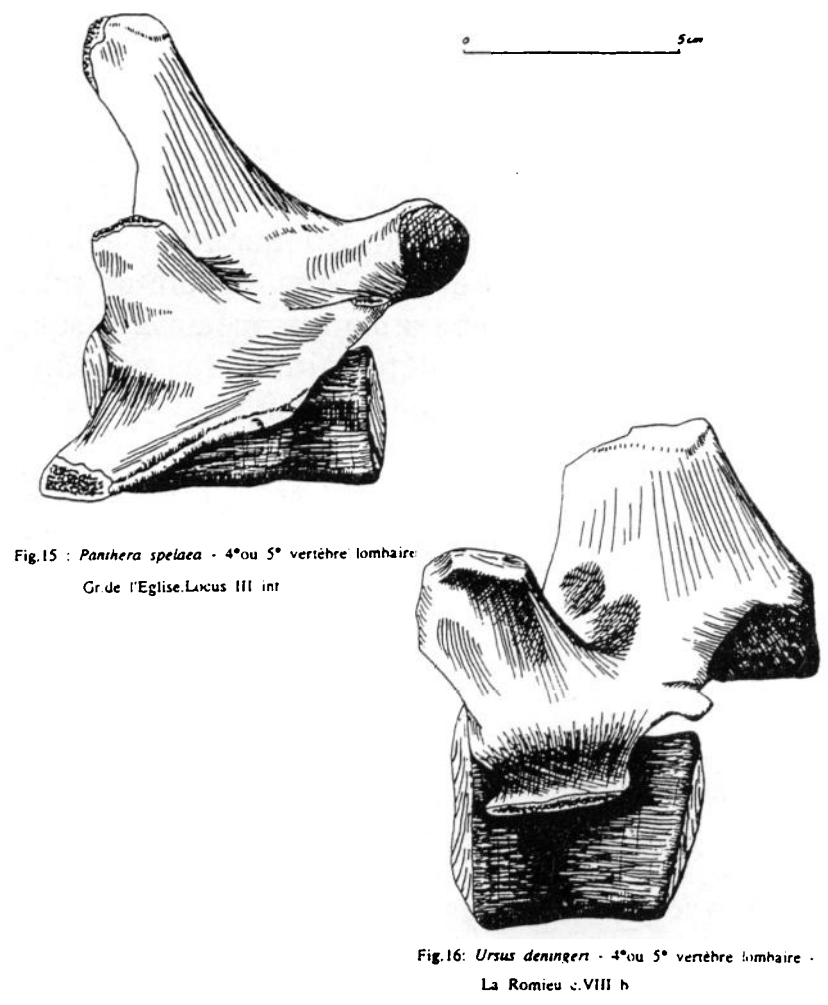

Nous l'avons attribuée à un Félin pour les raisons suivantes : chez les Félins, le corps vertébral des vertèbres lombaires est très allongé dans le sens crânio-caudal tandis qu'il est relativement court chez les Ursidés. La longueur crânio-caudale du corps vertébral de la pièce provenant de la Grotte de l'Eglise est de $64,5 \mathrm{~mm}$; le corps d'une vertèbre homologue d'Ursus deningeri de la Romieu (fouille F. Prat, couche $8 b$ ) n'atteint que $52,8 \mathrm{~mm}$. Le corps vertébral, en vue crâniale ou caudale, apparaît réniforme chez les Félins et non cordiforme comme chez les Ours.

Sur la vertèbre lombaire du Locus III inférieur de la Grotte de l'Eglise, l'apophyse quelque peu dété- riorée, inclinée en position crâniale et relativement haute, était vraisemblablement plutôt étroite dans le sens crânio-caudal ; elle s'implante nettement en avant des apophyses articulaires caudales. Les apophyses transverses sont inclinées en direction crânio-ventrale et les apophyses mamillaires se révèlent aiguës (Fig. 15). Chez l'Ours de la Romieu, l'apophyse épineuse est plus basse, plus large dans le sens crânio-caudal, et ne s'insère qu'en partie entre les apoyphyses articulaires caudales. D'autre part, les apophyses tranverses sont horizontales par rapport à l'axe de la colonne vertébrale, non inclinées en position ventrale, et les apophyses mamillaires sont globuleuses (Fig. 16).

\subsubsection{L'os coxal droit.}

Il est essentiellement constitué des régions iliaque et acétabulaire. La branche horizontale de l'ischion n'est qu'en partie conservée et la branche du pubis est fracturée à peu près à mi-longueur.

Cette pièce se distingue de son homologue chez les Ursidés par de nombreux détails anatomiques. Pour établir notre diagnose nous avons utilisé les os coxaux de Lion et d'Ours brun actuels de la collection de l'Institut du Quaternaire de l'Université de Bordeaux I.

Chez le Lion, la palette iliaque est pratiquement rectangulaire, le bord dorsal et le bord ventral étant parallèles. De plus elle ne se développe que dans un seul plan. Sa face latérale est creusée d'une fosse profonde et bien délimitée comme sur l'os que nous avions à déterminer et contrairement à ce que l'on constate chez l'Ours chez qui la palette de l'ilion est sub-triangulaire et amorce une torsion telle que l'épine ventro-crâniale est déjetée du côté latérodorsal.

Sur le spécimen de la Grotte de l'Eglise, l'épine iliaque dorso-caudale est à peine marquée comme chez le Lion ; elle développe au contraire un fort relief chez l'Ours.

Chez les félins au niveau de l'acétabulum, la corne postérieure de la surface semi-liunaire surplombe l'échancrure acétabulaire. On ne retrouve pas ce caractère chez l'Ours mais il est présent sur notre pièce fossile. Il s'agit donc bien d'un coxal de Felin.

Les dimensions de la vertèbre, de l'os coxal et des premières phalanges de la Grotte de l'Eglise sont plus fortes que celles des os homologues des grands Felins auxquels nous les avons comparées (Tabl. IV et V). Nous avons certainement affaire à Panthera spelaea. 


\begin{tabular}{|l|c|l|c|c|c|}
\hline $\begin{array}{l}\text { Mesures } \\
\text { en mm }\end{array}$ & \multicolumn{2}{|c|}{$\begin{array}{c}\text { P.spelaea } \\
\text { Gr. de l'Eglise }\end{array}$} & $\begin{array}{r}\text { P.spelaea } \\
\text { Gerde } \\
\text { CLOT 1980 }\end{array}$ & $\begin{array}{c}\text { P.spelaea } \\
\text { L'Arago } \\
\text { CREGUT 1979 }\end{array}$ & $\begin{array}{r}\text { P.leo } \\
\text { actuel }\end{array}$ \\
\hline HT & 62,5 & 61,5 & 52,9 & 68,3 & 48,0 \\
\hline DAP prox. & 22,5 & 20,0 & 18,7 & 22,9 & 16,0 \\
\hline DT prox. & 29,0 & 27,5 & 22,2 & 26,4 & 18,5 \\
\hline DAP dist. & 16,5 & 16,0 & 13,2 & 21,6 & 12,0 \\
\hline DT dist. & 21,0 & 20,0 & 17,4 & 17,0 & 15,0 \\
\hline DAP méd. & 14,0 & 14,0 & 12,2 & 18,2 & 10,5 \\
\hline DT méd. & 20,0 & 20,0 & 14,2 & 14,7 & 12,5 \\
\hline
\end{tabular}

Tableau IV : Panthera spelaea et Panthera leo -

Dimensions des premières phalanges

\begin{tabular}{|l|c|c|}
\hline $\begin{array}{l}\text { Mesures } \\
\text { en mm }\end{array}$ & $\begin{array}{l}\text { Panthra spelaea } \\
\text { Gr. de l'Eglise }\end{array}$ & $\begin{array}{l}\text { Panthera leo } \\
\text { actuel }\end{array}$ \\
\hline DAP cavité cotyloïde & 60 & 44 \\
\hline DT cavité cotyloïde & 54 & 41 \\
\hline Longueur de l'os iliaque & 200 & 146 \\
\hline $\begin{array}{l}\text { Diamètre verticale de la } \\
\text { colonne de l'ilion }\end{array}$ & 64 & 43 \\
\hline $\begin{array}{l}\text { Diamètre verticale de la } \\
\text { palette de l'iliaque }\end{array}$ & 80 & 68 \\
\hline
\end{tabular}

Tableau V : Panthera spelaea et Panthera leo -

Dimensions de l'os coxal.

\subsection{Lynx sp}

Une rotule (L. pales 1971), mesurant $24 \mathrm{~mm}$ de haut, $17 \mathrm{~mm}$ de large et $10 \mathrm{~mm}$ d'épaisseur, représente le genre Lynx dans le gisement. L'allongement de sa partie distale et sa faible épaisseur permet de distinguer cet os de la rotule du Loup.

\subsection{Vulpes ou Alopex.}

Une canine supérieure quelque peu usée et une vertèbre caudale provenant du Locus III inférieur indique un Renard sans que nous puissions dire s'il s'agit de Vulpes vulpes, le Renard commun, ou de Alopex lagopus, le Renard polaire. Selon A. Clot (1980, p. 129), l'étude des canines ne permet pas de distinguer ces deux genres, ce qui n'est tout à fait vrai que dans le cas où l'on ne dispose pas de dents intactes.

\subsection{Les Ursidés.}

Parmi les vestiges d'Ours recueillis par le Docteur Malenfant, nous avons reconnu trois espèces d'ursidés : Ursus deningeri, Ursus arctos et Ursus spelaeus, représentés respectivement par 110,6 et 7 vestiges osseux.

Ces pièces relativement nombreuses méritent une étude exhaustive qui ne pourrait entrer dans le cadre de cet article. Nous nous sommes limités ici à identifier les espèces présentes en utilisant les documents particulièrement significatifs, représentés surtout par les dents jugales.

\subsubsection{Ursus deningeri. :}

- Le fragment de maxillaire supérieur droit :

Cet os, outre la canine, porte les $\mathrm{P} 4, \mathrm{M} 1$ et M2 mais aussi une P3. Cette dent de petites dimensions, constante 
chez l'Ours étrusque du Pléistocène ancien, n'est constituée que d'un seul denticule. Selon F. Prat et $\mathrm{Cl}$ Thibault (1976) elle s'observe plus rarement chez Ursus spelaeus que chez Ursus deningeri. Chez Ursus arctos, $M$. Couturier (1954) signale qu'elle est relativement bien développée et souvent associée à une P2.

La présence de cette $\mathrm{P} 3$ et sa morphologie indiqueraient Ursus deningeri. Nous verrons ci-après que la morphologie de la P4 confirme cette détermination.

* Les dents jugales :

-4"prémolaire supérieure : (Fig. 17, D)

Sur les P4 trouvées isolées dans le Locus III inférieur et celle appartenant au fragment de maxillaire dont il vient d'être question, les crêtes du paracône et métacône réalisent un angle obtus et le deutérocône, indivis, est nettement séparé du métacône par un sillon profond. Cette disposition est bien caractéristique d'Ursus deningeri.
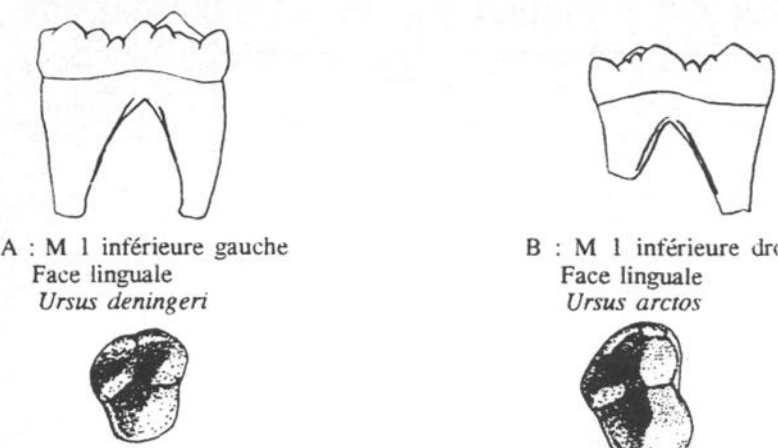

B : M 1 inférieure droite Face linguale Ursus arctos Ursus arctos

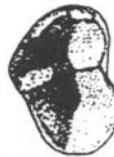

C : P 4 supérieure droite Face occlusale Ursus spelaeus
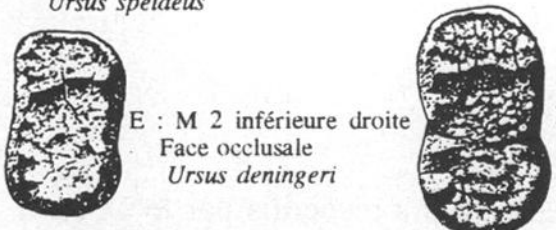

D : P 4 supérieure droite Face occlusale Ursus deningeri

Fig. 17 : Dents d'Ursidés - Grotte de l'Eglise.Locus III inf. - $4^{\text {me }}$ prémolaire inférieure :

La seule P4 provenant du Locus III inférieur est légèrement usée et sa racine est brisée au niveau de la couronne. Cependant elle possède des caractères morphologiques qui la rapprochent d'Ursus deningeri. Le protoconide est quelque peu décalé du côté mésial et de petits tubercules secondaires sont présents en position mésiale, mésiolinguale et disto-linguale sans toutefois être aussi développés que chez Ursus spelaeus.

$-1^{n}$ molaire inférieure : (Fig. 17, A)

La seule M1 de la collection montre un entoconide constitué de trois denticules dont la hauteur augmente dans le sens mésio-distal. D'autre part, le paraconide est dirigé verticalement. Ces caractères nous invitent à rap- porter sans la moindre hésitation cette dent à l'Ours de Deninger.

$2^{m \times}$ molaire inférieure : (Fig. 17, E)

7 échantillons.

Seulement 5 dents sont suffisamment bien conservées pour permettre une étude morphologique. Elles représentent toutes une légère constriction vestibulo-linguale entre le talonide et trigonide, ce qui les distingue des M2 d'Ursus arctos qui ne montrent pas de resserrement. De petits tubercules secondaires se développent, sur trois d'entre elles, entre le protoconide et le métaconide. L'hypoconide est souvent indivis mais sur certaines dents il commence à se dédoubler. D'une manière générale, ces molaires sont moyennement tuberculées et, par leurs caractères morphologiques, indiqueraient surtout Ursus deningeri.

\subsubsection{Ursus spelaeus.}

- Dents jugales :

- $4^{\text {mex }}$ prémolaire supérieure : (Fig. 17, C)

La crête du paracône et la crête du métacône de cette dent, sont sensiblement alignées et tendent à s'unir. Le deutérocône est pratiquement relié au métacône par une crête, comme cela se voit chez Ursus spelaeus.

$-2^{\mathrm{mm}}$ molaire inférieure : (Fig. 17, F)

Cette dent de grande taille (diamètre mésio-dista): $34,7 \mathrm{~mm}$ et diamètre vestibulo-lingual : $20,5 \mathrm{~mm}$ ) présente une constriction au centre de la couronne et un fort degré de tuberculisation. On remarque en particulier que de petits tubercules secondaires séparent le protoconide du métaconide.

Ces détails anatomiques plaident en faveur d'une attribution à Ursus spelaeus.

\subsubsection{Ursus arctos.}

-1* molaire inférieure : (Fig. 17, B)

Cette dent est de petites dimensions : son diamètre mésio-distal égale $27 \mathrm{~mm}$ et le diamètre vestibulolingual $13,9 \mathrm{~mm}$. L'entoconide est précédé mésialement par deux petits denticules, le paraconide est sensiblement vertical et la surface occlusale est parcourue par un sillon axial net. Il s'agit là d'une morphologie qui nous invite à attribuer cette molaire à l'Ours brun.

- Le talus (Fig.18) 


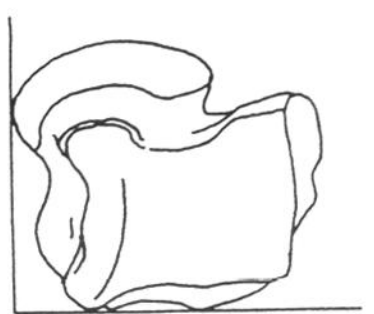

Fig.18: Talus droit d' Ursus arctos . en vue dorsale. dans un angle droit selon les conventions définies par J.ALTUNA 1973.

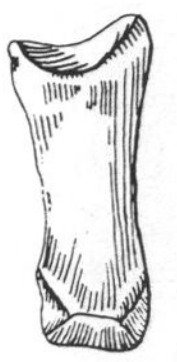

Ursus arctos Gr.de l'Eglise Locus III int.

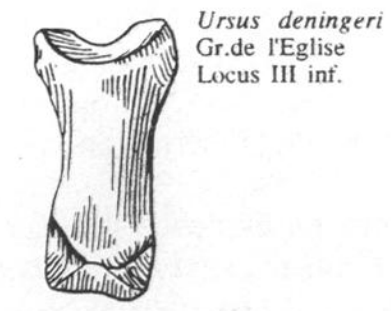

Ursus deningeri

La Romieu c.VIII h

H $14 n^{\circ} 609$
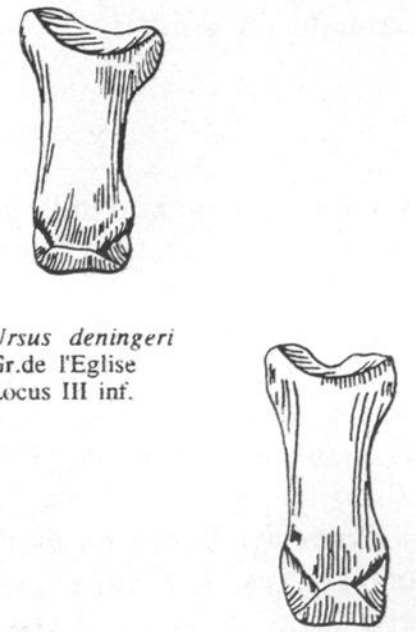

Ursus spelaeus Echenoz c.1la H $5 n^{\circ} 48$ ?
Fig.19: Phalange proximale du doigt I - Vue dorsale.

L'os étant examiné en vue dorsale, lorsque l'on inscrit sa partie proximale à l'intérieur d'un angle droit de façon que les deux points les plus proximaux du talus viennent au contact d'une des branches de l'angle, la tête articulaire est alors tangente à l'autre branche. Ce caractère serait propre à Ursus arctos (J. Altuna 1973).

- La phalange proximale du doigt I (Fig. 19).

Les pièces qui nous ont servi de terme de comparaison proviennent pour Ursus deningeri du Locus II inférieur de la Grottre de l'Eglise (H. Laville et al. 1972) et du gisement de Nauterie (F. prat et Cl. Thibault, 1976), pour Ursus spelaeus du gisement d'Echenoz-La-Méline (Jura) (Fouilles Campy). Malgré le faible échantillonnage, on remarque que cette phalange est nettement plus longue alors que son diamètre antéro-postérieur est à peu près identique à celui des autres pièces (tabl. VI) (A. Dubois et al. 1933). Il en résulte que l'indice de robustesse de cet os $(25,7)$ est plus faible que celui des phalanges correspondantes des autres Ursidés (30,4 pour Ursus deningeri de la Romieu et 29,4 pour Ursus spelaeus d'Echenoz). (J. Chagneau).

\section{Conclusion :}

Les trois espèces d'Ours seraient donc représentées dans l'ensemble faunique du Locus III inférieur de la Grotte de l'Eglise. Nous reviendrons plus loin sur les implications de la présence des deux Ours Ursus deningeri et Ursus spelaeus qui entrent tous dans la lignée de l'Ours des cavernes

\subsection{Rupicapra rupicapra ssp.}

Le Chamois n'est représenté dans le Locus III inférieur que par un scaphoïde droit. Cet os est

comparable à son homologue chez le Bouquetin, mais la présence d'un petit mamelon sur la face proximale, en position centrale, permet de l'en distinguer (Slott-Moller 1987).

Comme le Bouquetin, le Chamois est une espèce montagnarde qui s'est réfugiée en plaine, à plusieurs moments du Pléistocène, devant l'extension des glaciers. On le signale en Aquitaine à partir de la glaciation de Mindel (Camp de Peyre). Comme celle du Bouquetin, sa présence dans des régions non montagneuses indique l'instauraion de conditions climatiques froides, (F. prat 1966).

\subsection{Dicerorhinae indét.}

Une première phalange d'un doigt latéral d'une patte postérieure provenant du Locus III inférieur indique la sous-famille des Dicerorthinae, mais nous ne pouvons préciser s'il s'agit du genre Dicerorhinus ou du genre Coelodonta.

\subsection{Oryctolagus cuniculus grenalensis.}

E. Donard (1982) rapporte les formes quaternaires de Lapin du Sud de la France à une seule espèce, Oryctolagus cuniculus, mais distingue trois sousespèces pléistocènes :

- Oryctolagus cuniculus lunellensis, caractérisé par une taille moyenne, représenté dans le gisement de Lunel-Viel (Hérault) daté de l'interglaciaire Mindel-Riss.

- Oryctolagus cuniculus grenalensis, défini à partir de restes recueillis dans les couches rissiennes de Combe-Grenal (Dordogne), vraisemblablement représenté dans les couches rissiennes du Pech de l'Aze II, ainsi que dans divers gisements du Würm ancien. C'est une forme d'assez grande taille.

- Oryctolagus cuniculus huxleyi, de petite taille apparaît dans le sud-ouest de la France à la fin du Würm récent. 


\begin{tabular}{|l|c|c|c|c|}
\hline $\begin{array}{l}\text { Mesures } \\
\text { en mm }\end{array}$ & $\begin{array}{l}\text { Ursus arctos } \\
\text { Gr. de l'Eglise }\end{array}$ & $\begin{array}{l}\text { Ursus deningeri } \\
\text { Gr.de l'Eglise }\end{array}$ & $\begin{array}{l}\text { Ursus deningeri } \\
\text { La Romieu c.8 }\end{array}$ & $\begin{array}{l}\text { Ursus spelaeus } \\
\text { Echenoz c.11 }\end{array}$ \\
\hline H.T. & 51,7 & 40,9 & 43,0 & 43,5 \\
\hline D.A.P. méd & 13,3 & 11,8 & 13,1 & 12,8 \\
\hline DAP méd *100 & 25,7 & 28,8 & 30,4 & 29,4 \\
\hline \multicolumn{1}{c|}{ HT } & & & & \\
\hline
\end{tabular}

Tableau VI : Ursus arctos, Ursus deningeri, Ursus spelaeus -

Dimensions de la phalange proximale du doigt $I$.

Ces formes, relativement éloignées dans le temps et bien individualisées du point de vue biométrique, constitueraient donc d'assez bons repères chronologiques.

Le Lapin est représenté dans le Locus III inférieur de la Grotte de l'Eglise par 21 restes osseux.

Nous avons effectué quelques mesures utilisant la méthode employée par E. Donard. Les dimensions des deux extrémités distales de tibia et des trois calcanéums (Fig. 20) provenant du locus III inférieur de la Grotte de l'Eglise sont comparables à celles des os homologues de la sous-espece Oryctolagus cuniculus grenalensis. Ceci permettrait de situer chronologiquement la formation qui a livré ces os à l'intérieur de la longue période comprenant le complexe rissien, l'interglaciaire Riss/Würm et le Würm ancien.

\section{CONCLUSIONS}

La faune du Locus III inférieur de la Grotte de l'Eglise présente des caractères particuliers liés notamment aux Caprinés : dans les autres locus de la caverne, le Capriné présent est un Thar, dans le Locus III que nous étudions c'est un Bouquetin. Celui-ci est en outre beaucoup mieux représenté que dans tout autre gisement du Périgord ; 36,62 \% des vestiges lui sont attribuables alors que généralement dans les autres sites ce pourcentage ne dépasse pas 5 \% (F.Delpech 1983 et J.L. Guadelli 1987).

Les os de Bouquetin sont presque tous entiers et très bien conservés. Il est vrai qu'il n'y a aucun indice d'occupation humaine : les Bouquetins sont tombés dans une diaclase ayant joué le rôle de piège naturel. De plus les nombreux éléments du carpe, dont les surfaces articulaires compliquées permettent des remontages, ne proviennent vraisemblablement que de quelques sujets ce qui nous incite à penser que cette diaclase n'a piégé que très peu d'individus (le nombre minimum d'individus : NMI calculé à partir des talus droits est égal à 4).

En ce qui concerne la détermination de l'origine des vestiges de Carnivores, nous serons plus prudents, surtout à propos des Ours qui ont pu occuper les lieux au moment de l'hibernation.

Nous avons mis en évidence l'existence possible, entre autres, de Panthera spelaea, d'Ursus deningeri, et d'Ursus spelaeus. La présence de ces deux demiers pose le probleme de l'origine stratigraphique de tous ces ossements. On sait que dans la lignée de l'Ours des cavernes Ursus deningeri est l'ancêtre d'Ursus spelaeus. Nous insisterons pourtant sur le fait que tous les os, quel que soit le groupe auquel ils appartiennent, ont une même patine, une même couleur jaune pâle, qui les distinguent de tous les documents ostéologiques provenant des autres locus, ce qui pourrait être un argument en faveur de l'hypothèse selon laquelle les pièces du Locus III inférieur se trouvaient dans la même formation. Ursus deningeri est un ursidé considéré comme anté-würmien, Ursus spelaeus, son descendant, aurait vécu au cours de la dernière glaciation. On connaît mal toutefois les Ours de la fin du Riss et du dernier interglaciaire. C'est au cours de cette période que s'est effectué le passage de l'espèce Ursus deningeri à l'espèce Ursus spelaeus. Il ne serait donc pas surprenant de rencontrer au sein d'une même population des individus présentant des caractères propres à l'une ou l'autre espèce. La P4 supérieure et la M2 inférieure que nous avons décrites et qui présentent des caractères spéléens pourraient être alors considérées comme des cas de variation individuelle assez marginale au sein d'une population d'Ursus deningeri.

Peut-on tenter de donner un âge à cette faune en supposant que ces divers composants sont contemporains? 
Oryctolagus cuniculus grenalensis, que nous avons identifié, peut dater d'une période comprenant le complexe rissien et le Würm ancien.

Ursus deningeri situerait l'ensemble antérieurement au Würm.

Le Bouquetin, inconnu en France avant le Riss III (stade 6) (F. Delpech et F. Prat 1980), ne manifeste sa présence en Périgord et, d'une manière plus générale, dans les régions de plaine à relief quelque peu tourmenté, que lors des stades glaciaires rigoueux ; Riss III, Würm ancien II et Würm récent.

Dés lors, dans le cas d'un échantillon homogène, il serait facile de conclure en retenant la fin de la période rissienne pour l'âge de notre matériel. Le Bouquetin était alors présent en Périgord et les Ours de la lignée spéléenne ne présentaient pas encore les caractères de la forme type du Würm ancien.
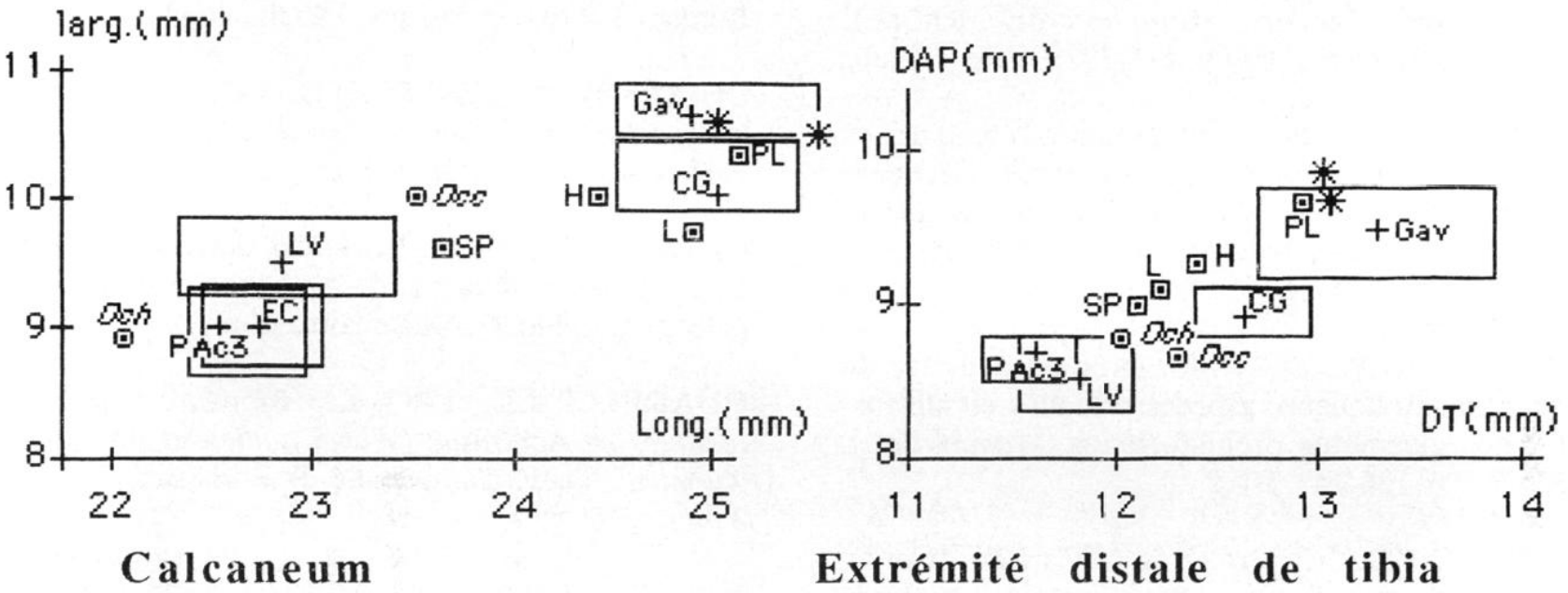

Calcaneum

LV : Lunel-Viel : Mindel/Riss

SP : Salpètre de Pompignan : Würm ancien II

CG : Combe-Grenal : Riss III

PAc3 : Pont d'Ambon, couche 3 : Azilien

L : Lazaret : Riss III

EC : El Gay : Epipaléolithique

PL : Pié-Lombard : Riss III

Occ : Oryctolagus cuniculus cuniculus : Actuel

H : Hortus : Riss III

Och : Oryctolagus cuniculus huxleyi : Actuel

Fig. 20 : Orytolagus cuniculus - Mensurations sur le calcaneum et le tibia 


\section{BIBLIOGRAPHIE}

ALTUNA, J., 1973. Hallazgos de Oso pardo (Ursus arctos, mammalia) en cuevas des Pais Vasco. Munibe, San Sebastian, t. 25, p. 121-170, 16 fig., 4 tabl., 3 ph., 21 pl.

BARONE, R. 1966. Anatomie comparée des Mammiferes domestiques.t. I : Ostéologie. Laboratoire d'anatomie. Ecole vétérinaire, Lyon. 81 p., très nombreuses fig.

BONIFAY, M.F., 1974-75. «Hemitragus bonali » Harlé et Stelshin, Caprinae de la Grotte de l'Escale (Saint-Estève-Janson, Bouche du Rhône). Quaternaria, Roma, XVIII:p. 215-302, 39 fig., 30 tab.

CHAGNEAU, J., 1985. Contribution à l'étude des os des extrémités des pattes d'Ursus deningeri romeviensis. Comparaison avec Ursus arctos et Ursus spelaeus. Bull.Soc. Anthropo., t. XX, n²-3, p. 61-107, 16 tab., 11 fig.

CHARLES, R.P., 1957. Morphologie dentaire du Thar et du Bouquetin, especes actuelles et sub-fossiles des gisements préhistoriques. Mammalia, t. $\mathrm{XXI}, \mathrm{n}^{\circ} 2$, p. 136-141, 2 fig.

CLOT, A., 1980. La Grotte de la Carrière (Gerde, Hautes-Pyrénées, France). Stratigraphie et paléontologie des carnivores. Thèse de troisième cycle, Université paul Sabatier, Toulouse, 2 tomes, 239 p., 114 fig., 131 tab., 1 pl.

CLOUET, M. et PFEFFER, P., 1979. Le Bouquetin d'Espagne : systématique et projet de réintroduction. Office National de la Chasse, Bulletin mensuel, $n^{\circ} 25$, mai.

COUTURIER, M., 1954. L'Ours brun. Couturier M.Ed. Grenoble, 903 p., 49 fig., 82 pl.

COUTURIER, M., 1962. Le Bouquetin des Alpes. Imprimerie Allier, Grenoble. 1564 p., nombreux tab., $141 \mathrm{pl}$.

CREGUT-BONNOURE, E., 1987. Caractères distincts du carpe et du tarse d'Hemitragus et de Capra (Mammalia Bovidea, Caprinea). Musée d'Anthrop. préhist. de Monaco. p. 161, 12 fig., Nombreux tab.

DELPECH, F. et PRAT, F., 1980. Les grands Mammiferes pleistocènes du Sud-Ouest de la France. In Problèmes de stratigraphie quaternaire en France et dans les pays limitrophes. Suppl. Bull. Asso. Fr. et Quat. N.S. Caen, n¹; p. 268-297, 3 fig., 1 tab.
DELPECH, F., 1983. Les faunes du Paléolithique supérieur dans le Sud-ouest de la France. Cahiers du Quarternaire. Ed. du CNRS, Paris. n6, 453 p., 147 tab., 6 pl.

DELPECH, F., 1988. Les grands Mammifères, à l'exception des Ursidés. In La grotte Vaufrey. Paléoenvionnements, chonologie, activités humaines. Jean-Philippe Rigaud éditeur. Mem. Soc. Préhist. fr., XIX, p. 213-289, 49 fig., 32 tab., 5 pl.

DONARD, E., 1982. Recherches sur les Léporidés quaternaires (Pléistocènes moyen et supérieur, Holocène). Thèse de troisième cycle $n^{\circ} 1764$ de Bordeux I. 2 tomes, 161 pp., 89 tab., 46 pl.

DUBOIS, A. et STEHLIN, H.G., 1933. La Grotte de Cotencher. Station moustérienne. Mém. de la Soc. Paléontologique Suisse. Vol. LII - LIII. 37 fig., 15 pl.

GRIGGO, C., 1991. Position taxinomique du Bouquetin de Malarnaud (Ariège) ; implications paléogéographiques. Quatenaire, ss. presse.

GUADELLI, J.L., 1987. Les zoocénoses préhistoriques en Aquitaine (Würm ancien et interstade würmien). Thèse d'Université de Bordeaux I. $n^{\circ} 148$, 3 tomes, $568 \mathrm{p}$.

LAVILLE, H., PRAT, F., THIBAULT, Cl., 1972. Un gisement à faune du Pléistocène moyen : la Grotte de l'Eglise, à Cénac-et-Saint-Julien (Dordogne). Quaternaria, Roma. t. XVI, pp. 71-119, 18 fig., 5 tab., 7 pl.

PALES, L.et LAMBERT, CH., Atlas ostéologique des Mammifères I). Les Membres. Herbivores : 84 pl., Carnivores : 48 pl. Ed. du CNRS, Paris.

PRAT, F., 1966. Les Caprinés. in R. Lavocat, Faunes et flores préhistoriques de l'Europe occidentale. Ed. Boubée, Paris, pp. 279-322, 3 fig., 19 pl.

PRAT, F. et THIBAULT, Cl. 1976. Le gisement de Nauterie à la Romieu (Gers). Fouilles 1967 à 1973. Nauterie I. Mém. Mus. Hist. Nat. Paris, c, 35,1-82, 35 fig., 13 tab., 7 pl.

SLOTT-MOLLER, R., 1987. Contribution à l'étude du basipode et de l'os malléolaire de l'Antilope saïga et de quelques autres Mammiferes. Soc. d'Anthrop. du Sud-Ouest. t. XXII, n², pp. 103-11, 13 tab., 10 fig.. 